\title{
AÇÕES DE AUTOCUIDADO NA SAÚDE ESCOLAR: REVISÃO INTEGRATIVA
}

\author{
SELF-CARE ACTIONS IN SCHOOL HEALTH: \\ AN INTEGRATIVE REVIEW
}

\section{ACCIONES DE AUTOCUIDADO EN SALUD ESCOLAR: UNA REVISIÓN INTEGRADORA}

\author{
Marjoriê da Costa Mendieta ${ }^{1}$ \\ Eliana Buss ${ }^{2}$ \\ Natália Rosiely Costa Vargas \\ Silvana Ceolin ${ }^{4}$ \\ Kamila Dias Gonçalves 5 \\ Rita Maria Heck ${ }^{6}$
}

Como citar este artigo: Mendieta MC, Buss E, Vargas NRC, Ceolin S, Gonçalves KD, Heck RM. Ações de autocuidado na saúde escolar: revisão integrativa. Rev baiana enferm. 2019;33:e31799.

Objetivo: analisar publicações científicas para conhecer as ações de autocuidado em saúde realizadas no âmbito escolar. Método: trata-se de estudo tipo revisão integrativa. A busca foi realizada na Literatura Latino-Americana em Ciências da Saúde (LILACS), no Scientific Electronic Library Online (SciELO) e no Public Medline (PubMed). Resultados: totalizaram a amostra da revisão integrativa 25 estudos, todos da base de dados PubMed, que possibilitaram compreender as ações de autocuidado em saúde no âmbito escolar, por meio de três temas principais: ações e programas escolares com foco em doenças, metodologia das ações nas escolas e papel da enfermagem no autocuidado escolar. Conclusão: a análise das publicações demonstrou que a maioria das ações de autocuidado em saúde nas escolas foca em patologias, poucos estudos utilizaram metodologias ativas e levaram em consideração o contexto sociocultural do escolar, o que, por consequência, pode tornar as ações menos efetivas em longo prazo.

Descritores: Serviços de Saúde Escolar. Enfermagem. Autocuidado. Cuidados de Enfermagem. Educação em Saúde. Promoção da Saúde.

Objective: to analyze scientific publications to know the self-care actions in health carried out at school. Method: this is an integrative review study. The search was performed in the Latin American Health Sciences Literature (LILACS), in the Scientific Electronic Library Online (SCiELO) and in Public Medline (PubMed). Results: the integrative review sample comprised 25 studies, all from the PubMed database, which made it possible to understand bealth self-care actions in the school environment, through three main themes: disease-focused school actions and programs, methodology of actions in schools and the role of nursing in school self-care. Conclusion: the analysis of the publications showed that most health self-care actions in schools focus on pathologies, few studies used active methodologies and took into account the sociocultural context of the student, which, consequently, can make the actions less effective in schools, in the long term.

Descriptors: School Health Services. Nursing. Self-care. Nursing Care. Health Education. Health Promotion.

\footnotetext{
Enfermeira. Doutora em Enfermagem. Pesquisadora independente. Pelotas, Rio Grande do Sul, Brasil. marjoriemendieta@gmail.com. https://orcid.org/ 0000-0002-6584-5560

2 Enfermeira. Mestre em Gestão de Políticas Públicas. Universidade Federal de Pelotas. Pelotas, Rio Grande do Sul, Brasil. https://orcid.org/0000-0003-3953-554X

3 Enfermeira. Mestre em Enfermagem. Universidade Federal de Pelotas. Pelotas, Rio Grande do Sul, Brasil. https://orcid.org/0000-0003-0081-9895

Enfermeira. Doutora em Enfermagem. Professora da Sociedade Educacional Três de Maio. Três de Maio, Rio Grande do Sul, Brasil. https://orcid.org/ 0000-000 I-6635-55I5

Enfermeira. Mestre em Ciências da Saúde. Universidade Federal de Pelotas. Pelotas, Rio Grande do Sul, Brasil. https://orcid.org/0000-0002-2632-2660

6 Enfermeira. Doutora em Enfermagem. Professora da Universidade Federal de Pelotas. Pelotas, Rio Grande do Sul, Brasil. https://orcid.org/0000-000 I-63 I 7-35I3
} 
Objetivo: analizar publicaciones cientificas para conocer las acciones de autocuidado en salud realizadas en el ámbito escolar. Método: es un estudio del tipo revisión integradora. La búsqueda se efectuó en la Literatura LatinoAmericana em Ciências da Saúde (LILACS), en Scientific Electronic Library Online (SciELO) y en Public Medline (PubMed). Resultados: 25 estudios totalizaron la muestra de la revisión integradora, todos de la base de datos PubMed, estudios que permitieron comprender las acciones de autocuidado en salud en el ámbito escolar, por medio de tres temas principales: acciones y programas escolares enfocados en enfermedades, metodología de las acciones en las escuelas y el rol de la enfermería en el autocuidado escolar. Conclusión: el análisis de las publicaciones demostró que la mayoría de las acciones de autocuidado en salud en las escuelas se enfoca en patologias, pocos estudios utilizaron metodologías activas y tuvieron en consideración el contexto sociocultural del ámbito escolar, lo que, por consecuencia, puede hacer que las acciones sean menos efectivas a largo plazo.

Descriptores: Servicios de Salud Escolar. Enfermería. Autocuidado. Cuidados de Enfermería. Educación en Salud. Promoción de la Salud.

\section{Introdução}

A educação escolar é considerada protagonista na formação do indivíduo. A escola é o espaço que acolhe o indivíduo para sua socialização, com semelhanças e diferenças, e onde o conhecimento se estrutura. Por conseguinte, pode ser um ambiente estratégico para o desenvolvimento de diversas ações que visem à melhoria das condições de saúde de crianças e adolescentes, por meio da promoção da saúde ${ }^{(1)}$, incentivando o desenvolvimento humano saudável e as relações construtivas e harmônicas, bem como o autocuidado.

O autocuidado caracteriza-se como uma função que permite às pessoas desempenharem atividades que visem à preservação da vida, saúde e bem-estar. Por ser uma capacidade de cuidar de si próprio, precisa ser incentivada e praticada desde a infância, para que se torne um contínuo na vida das pessoas ${ }^{(2)}$.

Nesse sentido, saúde e educação aproximam-se constantemente, quando se pensa nas condições de vida, constituindo um caminho para a conquista da qualidade de vida. Entretanto, há um grande desafio nessa interação ${ }^{(3)}$ : os profissionais da educação devem trabalhar sobre temas de saúde, e os profissionais da saúde devem utilizar práticas pedagógicas adequadas.

A escola propicia a aprendizagem do processo de educação em saúde por reunir, em um mesmo ambiente, pessoas em pleno crescimento e desenvolvimento. Para tanto, instigar o autocuidado, por meio da educação em saúde, requer dos profissionais o conhecimento aprofundado da temática, para possibilitar a construção desse saber sobre saúde voltado para a prática social do bem-estar e de uma vida saudável ${ }^{(3)}$.

Nessa construção, que deve ser conjunta com os profissionais de saúde, o escolar não deve ser visto como um ser passivo, que desenvolve ações repetitivas, mas, sim, um processador ativo de informações, isto é, faz as suas próprias descobertas. Para que isso ocorra de maneira efetiva, sugere-se que a abordagem seja contextualizada na realidade desse. Nessa proposta, quem ensina é um facilitador de aprendizagem e não apenas um transmissor de informações ${ }^{(4)}$.

Nesse contexto, propostas atuais, que envolvem a promoção da saúde na escola, demonstram que o diálogo baseado nas vivências dos estudantes é essencial. Para isso, é necessária uma abordagem que relacione a diversidade do contexto da comunidade escolar, que é constituída de sujeitos que possuem diferentes saberes $^{(1)}$. Em consequência, ressalta-se a importância e a necessidade de práticas pedagógicas ativas e inovadoras, que tenham como foco uma formação integral de cidadãos críticos e reflexivos, nos diferentes contextos de vida ${ }^{(5)}$. Entretanto, sabe-se que ações de saúde desenvolvidas historicamente na escola têm se centrado, muitas vezes, no olhar biomédico cujo foco está na doença ou na sua prevenção. Essa forma de pensar tem sido insuficiente para fazer da escola um espaço para produzir saúde ${ }^{(6)}$. 
Diante disso, reflete-se que, na atualidade, diante dos avanços, tanto na saúde como na educação, sobretudo no quesito científico, as práticas de educação em saúde na escola, especialmente quanto ao estímulo ao autocuidado, permanecem restritas às metodologias de ensino tradicionais e focam somente na doença.

Dessa maneira, o presente estudo teve como questão norteadora: "Quais ações de autocuidado em saúde são praticadas e/ou incentivadas na escola?". O objetivo deste artigo é analisar publicações científicas para conhecer as ações de autocuidado em saúde realizadas no âmbito escolar.

\section{Método}

Trata-se de estudo do tipo revisão integrativa, estratégia metodológica que permite a busca sistemática e abrangente, avaliação crítica e síntese de pesquisas relevantes sobre determinada temática $^{(7)}$. Desenvolveu-se esta revisão em seis etapas $^{(8)}$. Inicialmente, identificou-se o tema e selecionou-se a questão de pesquisa: Quais são as ações de autocuidado em saúde praticadas e/ou incentivadas na escola?

$\mathrm{Na}$ segunda etapa, estabeleceram-se os critérios para inclusão e exclusão de estudos. Os critérios para inclusão foram artigos científicos que tratassem a temática, publicados nos idiomas português, inglês ou espanhol, e publicados nos últimos 10 anos (2009-2018), visando obter os estudos mais recentes e relevantes. O critério de exclusão foi não ser artigo original de pesquisa.
Foram consultados os Descritores em Ciências da Saúde (DeCS), Serviços de Saúde Escolar e Autocuidado (ou School Health Services e Self Care), e o operador boleano "and". Destaca-se que foi utilizado o descritor "Serviços de Saúde Escolar", uma vez que não existe descritor para o termo "Saúde Escolar".

A busca foi realizada na Literatura Latino-Americana em Ciências da Saúde (LILACS), no Scientific Electronic Library Online (SciELO) e no Public Medline (PubMed). Na base de dados LILACS, realizou-se a busca mediante a utilização da "Pesquisa via Formulário iAH", com a busca por "palavras" e não por descritor de assunto, visto que, dessa maneira, alcançava-se maior número de estudos. Na SciELO, realizou-se a busca por "Todos os índices". No PubMed, a busca ocorreu pelos MeSH Terms. A busca e a análise das produções foram realizadas no período de janeiro a março de 2019.

As buscas foram efetuadas com os descritores em inglês e em português, a fim de identificar em qual idioma obter-se-iam maiores resultados. Entretanto, os descritores em inglês somente foram utilizados no PubMed, visto que, nas outras buscas, encontrava-se maior resultado com descritores em português.

Identificaram-se 223 estudos, dos quais 86 foram selecionados para leitura e análise por apresentarem os critérios de inclusão previamente estabelecidos. Para esse processo de seleção, utilizou-se as recomendações do Preferred Reporting Items for Systematic reviews and Meta-Analyses (PRISMA) (Figura 1) ${ }^{(9)}$. 
Figura 1 - Fluxograma da seleção dos estudos para a revisão integrativa

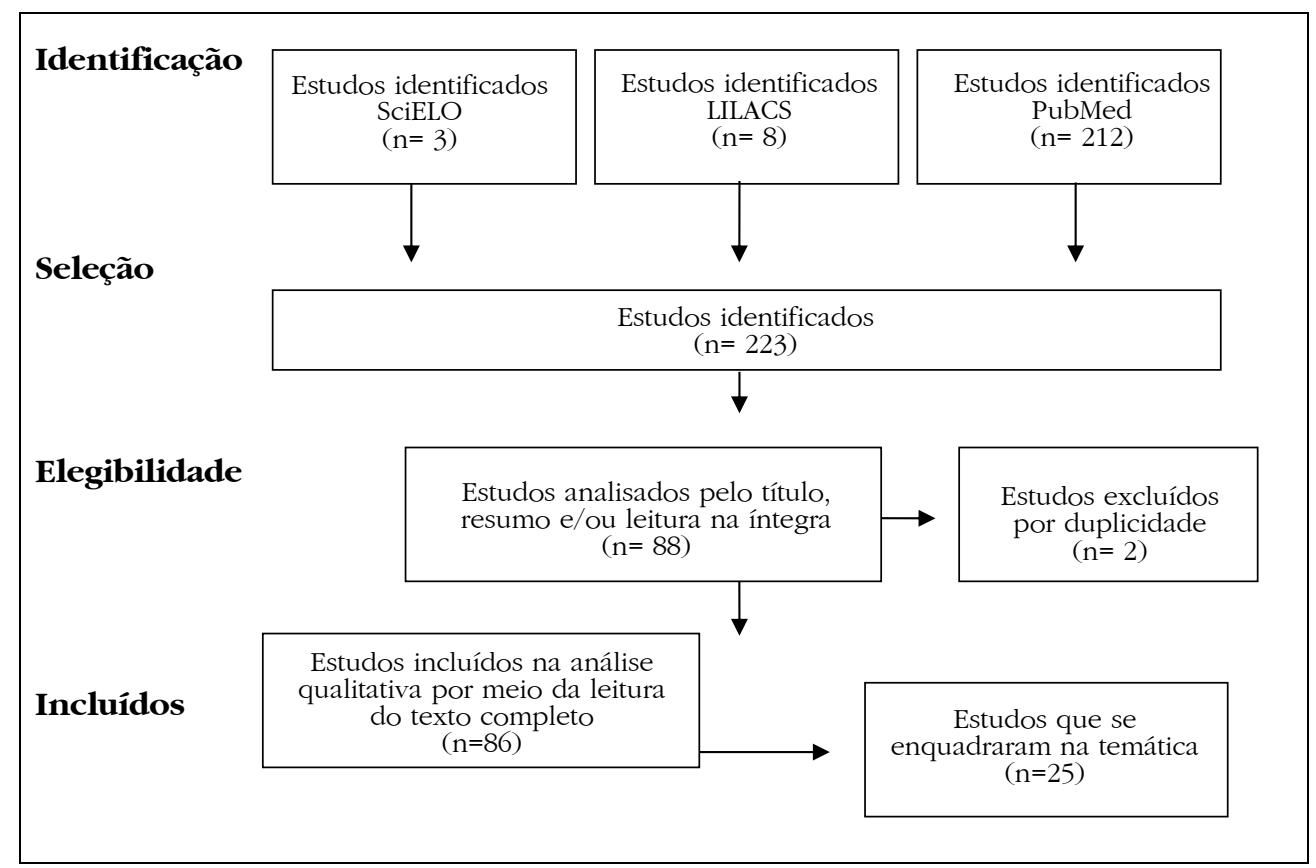

Fonte: Elaboração própria.

A terceira etapa consistiu na leitura dos 86 artigos selecionados na íntegra, para identificar se respondiam à questão de pesquisa. Após essa leitura, 25 estudos totalizaram a amostra dessa revisão integrativa, todos da base de dados PubMed, e fizeram parte da quarta etapa, que consistiu no preenchimento e avaliação do instrumento com os dados das publicações selecionadas. Esta avaliação foi realizada por quatro pesquisadoras. Para a coleta de dados dos artigos, elaborou-se um instrumento com as informações: biblioteca/base de dados, autores, título, temática principal, objetivo do estudo, metodologia, ano de publicação, periódico, local da pesquisa, principais resultados e nível de evidência.

A quinta etapa consistiu na discussão e interpretação dos resultados, seguida da sexta etapa, que apresentou as evidências encontradas.

\section{Resultados}

A amostra final desta revisão foi constituída por 25 estudos (Quadro 1), identificando-se três temas principais: Ações e programas escolares com foco em doenças; Metodologia das ações nas escolas; Papel da enfermagem no autocuidado escolar.

Quadro 1 - Produção científica relacionada às ações de autocuidado na saúde escolar no período de 2009 a 2018

(continua)

\begin{tabular}{|l|l|c|}
\hline Título do artigo e Ano & \multicolumn{1}{|c|}{ Objetivo } & $\begin{array}{c}\text { Nivel de } \\
\text { Evidência }\end{array}$ \\
\hline $\begin{array}{l}\text { Self-care management of type 1 diabetes } \\
\text { has improved in Swedish schools } \\
\text { according to children and adolescents. } \\
2017^{(10)}\end{array}$ & $\begin{array}{l}\text { Avaliar o apoio para autocuidado de } \\
\text { crianças com diabetes em horário escolar. }\end{array}$ & III \\
\hline $\begin{array}{l}\text { Effectiveness of a school-and community- } \\
\text { based academic asthma health education } \\
\text { program on use of effective asthma } \\
\text { self-care behaviors in older school-age } \\
\text { students. } \\
2015^{(11)}\end{array}$ & $\begin{array}{l}\text { Avaliar a eficácia de um programa de } \\
\text { edução para a saúde sobre asma. }\end{array}$ & II \\
\hline
\end{tabular}


Quadro 1 - Produção científica relacionada às ações de autocuidado na saúde escolar no período de 2009 a 2018

(continuação)

\begin{tabular}{|c|c|c|}
\hline Título do artigo e Ano & Objetivo & $\begin{array}{l}\text { Nivel de } \\
\text { Evidência }\end{array}$ \\
\hline $\begin{array}{l}\text { Effectiveness of a modified open airways } \\
\text { curriculum. } \\
2015^{(12)}\end{array}$ & $\begin{array}{l}\text { Aplicar um programa de educação sobre } \\
\text { asma, de forma condensada, e avaliar sua } \\
\text { eficácia. }\end{array}$ & III \\
\hline $\begin{array}{l}\text { Training at-risk youth to become diabetes } \\
\text { self-management coaches for family } \\
\text { members. } \\
2014^{(13)}\end{array}$ & $\begin{array}{l}\text { Avaliar o impacto de um programa de } \\
\text { saúde escolar sobre ações de autocuidado } \\
\text { para membros da família com diabetes. }\end{array}$ & III \\
\hline $\begin{array}{l}\text { The effectiveness of an integrated } \\
\text { multicomponent program for adolescent } \\
\text { smoking cessation in Taiwan. } \\
2014^{(14)}\end{array}$ & $\begin{array}{l}\text { Desenvolver e avaliar um programa de } \\
\text { cessação do tabagismo para estudantes } \\
\text { fumantes de escolas de ensino médio. }\end{array}$ & II \\
\hline $\begin{array}{l}\text { Evaluation of a pilot national online } \\
\text { asthma e-learning program for secondary } \\
\text { school students. } \\
2014^{(15)}\end{array}$ & $\begin{array}{l}\text { Avaliar um programa piloto on-line } \\
\text { à distância, para estudantes asmáticos. }\end{array}$ & III \\
\hline $\begin{array}{l}\text { Diabetes management in Swedish schools: } \\
\text { a national survey of attitudes of parents, } \\
\text { children, and diabetes teams. } \\
2014^{(16)}\end{array}$ & $\begin{array}{l}\text { Investigar atitudes entre crianças } \\
\text { e adolescentes com diabetes, seus pais } \\
\text { e sua equipe de saúde a respeito de } \\
\text { cuidados com diabetes na escola. }\end{array}$ & III \\
\hline $\begin{array}{l}\text { Establishing a model to assess the effects } \\
\text { of school support and self-care behaviors } \\
\text { on life satisfaction in adolescents with } \\
\text { type } 1 \text { diabetes in Taiwan. } \\
2013\end{array}$ & $\begin{array}{l}\text { Construir um modelo que avalie os efeitos } \\
\text { do apoio escolar e de comportamentos de } \\
\text { autocuidado de adolescentes com diabetes } \\
\text { tipo } 1 \text { em Taiwan. }\end{array}$ & III \\
\hline $\begin{array}{l}\text { Process and outcomes of school nurse } \\
\text { case management for students with } \\
\text { asthma. } \\
2014^{(18)}\end{array}$ & $\begin{array}{l}\text { Avaliar o impacto da enfermeira escolar } \\
\text { sobre o gerenciamento de saúde e o } \\
\text { impacto acadêmico para escolares com } \\
\text { asma. }\end{array}$ & III \\
\hline $\begin{array}{l}\text { Effects of coping-skills training in } \\
\text { low-income urban african-american } \\
\text { adolescents with asthma. } \\
2012^{(19)}\end{array}$ & $\begin{array}{l}\text { Determinar os efeitos de um programa } \\
\text { de treinamento de habilidades de } \\
\text { enfrentamento com adolescentes } \\
\text { afro-americanos com asma. }\end{array}$ & II \\
\hline $\begin{array}{l}\text { Peer-led education for adolescents with } \\
\text { asthma in Jordan: a cluster-randomized } \\
\text { controlled trial. } \\
2012^{(20)}\end{array}$ & $\begin{array}{l}\text { Determinar o impacto de um programa } \\
\text { de educação sobre saúde em escolares do } \\
\text { ensino médio com asma na Jordânia. }\end{array}$ & II \\
\hline $\begin{array}{l}\text { Medication use patterns among urban } \\
\text { youth participating in school-based } \\
\text { asthma education. } \\
2011^{(21)}\end{array}$ & $\begin{array}{l}\text { Avaliar se a participação em programa } \\
\text { de asma baseado na escola melhorou } \\
\text { a utilização de medicação para a asma } \\
\text { entre estudantes do ensino médio. }\end{array}$ & III \\
\hline $\begin{array}{l}\text { Improving the mental health, healthy } \\
\text { lifestyle choices, and physical health } \\
\text { of Hispanic adolescents: a randomized } \\
\text { controlled pilot study. } \\
2009^{(22)}\end{array}$ & $\begin{array}{l}\text { Avaliar a eficácia preliminar de um } \\
\text { programa de educação comportamental } \\
\text { e cognitivo com adolescentes. }\end{array}$ & II \\
\hline $\begin{array}{l}\text { Reducing adolescent use of harmful } \\
\text { legal products: intermediate effects of } \\
\text { a community prevention intervention. } \\
2009^{(23)}\end{array}$ & $\begin{array}{l}\text { Avaliar a intervenção preventiva para } \\
\text { reduzir o uso de substâncias nocivas } \\
\text { legalizadas entre estudantes de três } \\
\text { comunidades rurais do Alasca. }\end{array}$ & III \\
\hline
\end{tabular}


Quadro 1 - Produção científica relacionada às ações de autocuidado na saúde escolar no período de 2009 a 2018

(conclusão)

\begin{tabular}{|c|c|c|}
\hline Título do artigo e Ano & Objetivo & $\begin{array}{c}\text { Nível de } \\
\text { Evidência }\end{array}$ \\
\hline $\begin{array}{l}\text { Effects of a school-based weight } \\
\text { maintenance program for Mexican- } \\
\text { American children: results at } 2 \text { years. } \\
2010^{(24)}\end{array}$ & $\begin{array}{l}\text { Avaliar os resultados de um programa de } \\
\text { manutenção de peso baseada no estilo de } \\
\text { vida com crianças com sobrepeso em uma } \\
\text { escola em Houston, Texas. }\end{array}$ & II \\
\hline $\begin{array}{l}\text { Improving asthma management in the } \\
\text { elementary school setting: an education } \\
\text { and self-management pilot project. } \\
2018^{(25)}\end{array}$ & $\begin{array}{l}\text { Avaliar um programa de educação } \\
\text { e autoavaliação da asma, por meio de } \\
\text { uma parceria entre uma escola de ensino } \\
\text { fundamental e uma escola de enfermagem. }\end{array}$ & VI \\
\hline $\begin{array}{l}\text { A cluster randomized theory-guided oral } \\
\text { hygiene trial in adolescents - A latent } \\
\text { growth model. } \\
2017^{(26)}\end{array}$ & $\begin{array}{l}\text { Testar se as intervenções guiadas pela } \\
\text { teoria "Processo de Adoção da Precaução" } \\
\text { são mais eficazes que a instrução } \\
\text { odontológica convencional para mudar } \\
\text { a higiene bucal com adolescentes. }\end{array}$ & II \\
\hline $\begin{array}{l}\text { Self-efficacy theory-based intervention in } \\
\text { adolescents: a cluster randomized trial- } \\
\text { focus on oral self-care practice and oral } \\
\text { self-care skills. } \\
2017^{(27)}\end{array}$ & $\begin{array}{l}\text { Examinar a eficácia da educação } \\
\text { odontológica orientada pela teoria } \\
\text { da autoeficácia, para a melhoria do } \\
\text { autocuidado oral em adolescentes. }\end{array}$ & II \\
\hline $\begin{array}{l}\text { Enhancing asthma self-management in } \\
\text { rural school-aged children: a randomized } \\
\text { controlled trial. } \\
2016^{(28)}\end{array}$ & $\begin{array}{l}\text { Testar os efeitos de dois modos de } \\
\text { aplicação de uma intervenção educativa } \\
\text { sobre autocuidado da asma com crianças } \\
\text { em idade escolar que vivem em áreas } \\
\text { rurais. }\end{array}$ & III \\
\hline $\begin{array}{l}\text { A theory-guided school-based intervention } \\
\text { in order to improve adolescents' oral self- } \\
\text { care: a cluster randomized trial. } \\
2016^{(29)}\end{array}$ & $\begin{array}{l}\text { Avaliar a eficácia das intervenções } \\
\text { de higiene oral orientadas pela teoria } \\
\text { sociocognitiva em adolescentes. }\end{array}$ & II \\
\hline $\begin{array}{l}\text { The effectiveness of gain-versus loss- } \\
\text { framed health messages in improving } \\
\text { oral health in Iranian secondary schools: } \\
\text { a cluster-randomized controlled trial. } \\
2014^{(30)}\end{array}$ & $\begin{array}{l}\text { Examinar os efeitos de intervenções } \\
\text { baseadas na entrega de fôlderes sobre } \\
\text { comportamentos de autocuidado oral } \\
\text { e saúde entre adolescentes iranianos. }\end{array}$ & II \\
\hline $\begin{array}{l}\text { Effect of a school-based oral health } \\
\text { education programme on use of } \\
\text { recommended oral self-care for reducing } \\
\text { the risk of caries by children in Nigeria. } \\
2015^{(31)}\end{array}$ & $\begin{array}{l}\text { Avaliar o efeito de um programa de } \\
\text { educação em saúde bucal baseado } \\
\text { na escola sobre o uso de medidas de } \\
\text { autocuidado oral para reduzir a cárie. }\end{array}$ & II \\
\hline $\begin{array}{l}\text { Pediatric obesity and asthma quality of } \\
\text { life. } \\
2013^{(32)}\end{array}$ & $\begin{array}{l}\text { Relatar os efeitos da obesidade infantil } \\
\text { e da asma na qualidade de vida e o } \\
\text { enfrentamento e controle em adolescentes } \\
\text { afro-americanos de baixa renda com asma. }\end{array}$ & II \\
\hline $\begin{array}{l}\text { Increasing availability to and ascertaining } \\
\text { value of asthma action plans in } \\
\text { schools through use of technology and } \\
\text { community collaboration. } \\
2013^{(33)}\end{array}$ & $\begin{array}{l}\text { Avaliar as respostas de enfermeiras } \\
\text { das escolas ao Plano de Ação para } \\
\text { a Asma eletrônico, quanto à eficiência, } \\
\text { autoeficácia e impacto do projeto para } \\
\text { o autocuidado da asma. }\end{array}$ & $\mathrm{VI}$ \\
\hline $\begin{array}{l}\text { An intervention to increase high school } \\
\text { students' compliance with carrying auto- } \\
\text { injectable epinephrine: a MASNRN study. } \\
2012^{(34)}\end{array}$ & $\begin{array}{l}\text { Testar a eficácia das intervenções } \\
\text { da enfermeira da escola para } \\
\text { a disponibilidade de epinefrina } \\
\text { autoinjetável. }\end{array}$ & II \\
\hline
\end{tabular}

Fonte: Elaboração própria. 
Identificou-se que a maioria das publicações (24\%) é do ano de 2014. Dos 25 estudos, os periódicos com maior número de publicações foram o International Journal of Pediatric Dentistry com 3 estudos (12\%), seguido do The Journal of School Nursing, Journal of School Health e The Journal of Nursing Research com 2 estudos cada um (8\%). Quanto aos países de origem dos estudos, os que tiveram mais publicações foram os Estados Unidos da América (EUA), com 14 publicações (56\%); seguido da China, Suécia e Lituânia, com 2 publicações cada (8\%); e Nigéria, Irã, Canadá, Jordânia e Irlanda, com 1 publicação cada (4\%).

Os estudos tiveram diferentes focos para suas ações nas escolas, entretanto, destaca-se a asma como tema de 11 estudos, totalizando 44\%, seguido de saúde bucal, com 5 estudos (20\%) e diabetes, com 4 estudos (16\%).

A qualidade metodológica dos estudos concentrou-se nos Níveis de Evidência ${ }^{(35)}$ II (52\% dos estudos), III (40\% dos estudos) e VI ( $8 \%$ dos estudos).

\section{Discussão}

A leitura dos 25 estudos resultou na identificação de três temas: Ações e programas escolares com foco em doenças; Metodologia das ações nas escolas; e Papel da enfermagem no autocuidado escolar, os quais serão apresentados a seguir.

\section{Ações e programas escolares com foco em doenças}

$\mathrm{Na}$ análise dos estudos, constatou-se que a maioria teve como foco o autocuidado com alguma patologia específica. Dos 25 estudos, $11^{(11-12,15,18-21,25,28,32-33)}$ abordaram escolares asmáticos - destes, 9 foram realizados nos EUA ${ }^{(11-}$ 12,18-19,21,25,28,32-33) - e 10 avaliaram a eficácia de diferentes programas, na escola, para o controle dessa patologia ${ }^{(11-12,15,19-21,25,28,32-33)}$.

O estudo realizado no Texas (EUA) abordou estudantes em transição do ensino fundamental para o ensino médio, no qual foi implementado um programa - Staying Healthy-Asthma Responsible and Prepared (SHARP) - para promover o autocuidado da asma. Após o programa, houve aperfeiçoamento na gestão de episódios de asma, além de melhoria de comportamentos para a promoção da saúde ${ }^{(11)}$.

Outro estudo utilizou o programa The Open Airways, de maneira condensada, a fim de verificar sua efetividade comparada ao programa original - em vez de 6 sessões de 40 minutos, foram realizadas sessões de 20 minutos. Foram incluídas crianças de 8 a 12 anos, e verificou-se que o programa, na versão modificada, assim como em sua versão original, era eficaz para melhorar o conhecimento sobre como gerir disparadores e sintomas de asma, bem como para aperfeiçoar a técnica de inalação ${ }^{(12)}$.

O programa Teen Educational Asthma Management (TEAM) foi utilizado em duas pesquisas de autores em comum ${ }^{(19,32)}$. Esse programa inclui três etapas principais: educação sobre a asma, treinamento de habilidades para enfrentamento e visitas de enfermagem para reforço $^{(32)}$. Um desses estudos foi desenvolvido com adolescentes de 13 a 19 anos de uma escola urbana. A ausência motivada pela asma diminuiu após a implementação do programa. Além disso, observou-se maior conhecimento sobre o autocuidado com a asma ${ }^{(19)}$. O segundo estudo, que utilizou o TEAM, para avaliar o impacto na qualidade de vida de adolescentes portadores de asma e obesidade, concluiu que a obesidade, apesar de afetar a qualidade de vida de maneira negativa, não interferiu no enfrentamento e controle da asma ${ }^{(32)}$.

O Programa Triple $A$ foi o escolhido no estudo realizado na Jordânia, com estudantes adolescentes portadores de asma. Para o recrutamento de estudantes foi utilizado o questionário autoaplicado Estudo Internacional de Asma e Alergia na Infância (ISAAC), instrumento validado para avaliar a asma. Os autores concluíram que a saúde desses asmáticos foi significativamente melhorada após a intervenção ${ }^{(20)}$. Outro estudo, que também utilizou o ISAAC para recrutar estudantes do ensino médio de escolas urbanas, implementou o programa Kickin'Asthma, 
a fim de melhorar a utilização de medicamentos para asma e, efetivamente, constatou melhorias importantes no uso da medicação ${ }^{(21)}$.

Além desses, outros estudos envolviam a asma como tema principal, como o programa on-line com estudantes de 15-16 $\operatorname{anos}^{(15)}$, e dois estudos $^{(25,33)}$ baseados no Plano de Ação para a Asma - Asthma Action Plans (AAP) - que consistem em planos de autocuidado para controle e prevenção de crises de asma. Em um deles, desenvolvido com escolares do ensino fundamental, estudantes de enfermagem realizaram atividades educativas para identificar os AAP e constataram o aumento do conhecimento dos estudantes após as atividades ${ }^{(25)}$. A outra pesquisa desenvolveu um portal eletrônico para facilitar a transferência do AAP para a escola. Enfermeiras escolares avaliaram o portal e o consideraram um aliado na assistência à saúde escolar, e que proporcionaria aumento da comunicação entre profissionais da saúde, familiares e escola ${ }^{(33)}$.

Todos os estudos envolviam a asma, com a justificativa de que ela ocasionava falta à escola e prejudicava o rendimento escolar. Dessa maneira, pôde-se inferir que esses diferentes tipos de programas consistiam em alternativas eficazes para trabalhar educação em saúde com asmáticos na escola e que poderiam impactar na melhoria do autocuidado e redução das ausências à escola devido às crises.

Além da asma, outros estudos implementaram atividades para trabalhar a diabetes com os escolares. Estudo realizado em São Francisco (EUA) destacou-se, pois buscou formar adolescentes saudáveis para serem treinadores de autocuidado para membros diabéticos da família, incentivando, assim, o autocuidado e a autonomia de outras pessoas ${ }^{(13)}$. No entanto, o método dessa formação não foi detalhado, ficando o questionamento acerca da coerência dessas ações em relação às práticas já realizadas por essas pessoas no âmbito familiar. Salienta-se assim, a importância de atividades coerentes com a realidade sociocultural dos escolares, para que se tornem efetivas.
Além desse estudo, outros três também tiveram como foco a diabetes ${ }^{(10,16-17)}$. Dois, investigaram as ações de escolares diabéticos e de seus pais sobre cuidados com diabetes na escola ${ }^{(10,16)}$, demonstrando que há deficiências no apoio da gestão de autocuidado escolar. Outro, realizado em Taiwan, buscou o estabelecimento de um modelo para avaliar os efeitos do apoio escolar e de comportamentos de autocuidado sobre a satisfação com a vida de adolescentes diabéticos tipo $1^{(17)}$. O programa consistiu em aplicar questionários, escalas de autocuidado e testes de glicose, com vistas a proporcionar o gerenciamento da diabetes e o apoio escolar ${ }^{(17)}$.

Três estudos tiveram como foco o sobrepeso $^{(24)}$, o tabagismo ${ }^{(14)}$ e a saúde bucal $^{(30)}$. O estudo realizado com crianças de 10 a 14 anos em uma escola no Texas avaliou um programa de manutenção de peso. O acompanhamento foi realizado durante dois anos e, no final, observou-se melhoria não somente no peso, com a diminuição do Índice de Massa Corporal (IMC), mas também redução da taxa de colesterol e triglicerídeos ${ }^{(24)}$.

Um programa para desenvolver a cessação do tabagismo para estudantes fumantes do ensino médio foi realizado em Taiwan. Ao final da pesquisa, um teste de urina realizado pelos participantes, para confirmar a abstinência, demonstrou a eficácia estatística do programa ${ }^{(14)}$.

Relacionado à saúde bucal, pesquisa avaliou uma intervenção com escolares do ensino médio, mediante a entrega de dois tipos de fôlderes informativos: um com enquadramento de perda, que destacava os riscos de não cuidar da saúde bucal, e outro, no ganho, com a exposição de benefícios. Após avaliação baseada na opinião e nas atitudes referidas pelos estudantes, os autores constataram que os fôlderes com destaque para os riscos foram mais eficazes para encorajar o autocuidado em saúde bucal ${ }^{(30)}$.

Todos os programas e as atividades colocados em prática nessas pesquisas foram considerados eficazes para os escolares, podendo ser uma possibilidade de replicação em diferentes locais. No entanto, percebe-se que essas 
ações são predominantemente direcionadas à doença e não à saúde. É inquestionável a sua relevância, no entanto, o que se quer enfatizar é que a maioria dos estudos incluídos nesta revisão integrativa, apesar de buscar ações de autocuidado em saúde, resultou em trabalhos que visavam o autocuidado diante de uma patologia já estabelecida, ignorando a promoção da saúde, focando somente na prevenção de doenças e agravos.

A maioria desses estudos justificou que a escolha deveu-se à ausência de crianças/adolescentes na escola associados à patologia, com impacto no processo de ensino aprendizagem.

Estudo realizado em uma escola pública de Salvador, Bahia, Brasil, identificou que há diferentes aspectos sociais e de saúde relacionados à reprovação escolar, como iniciação sexual na adolescência, gravidez na adolescência, vivência de violência psicológica e necessidade de conciliar a escola com o trabalho para contribuir no sustento da família ${ }^{(36)}$.

Além desses fatores, a prática pedagógica das ações de saúde na escola também necessita ser coerente, de modo que a aprendizagem torne-se significativa para o escolar. Este aspecto metodológico das atividades será abordado a seguir.

\section{Metodologia das ações nas escolas}

Os estudos abordaram os escolares de diferentes maneiras, no que se refere à metodologia de aprendizagem. Dessa maneira, alguns se destacaram por detalharem os recursos utilizados no desenvolvimento dessas atividades, tais como discussões, histórias, jogos, folhetos e vídeos ${ }^{(12,19,28)}$. Entretanto, na maioria dos estudos, este esclarecimento estava ausente.

O programa The Open Airways, que consiste em seis sessões educativas de 40 minutos, foi utilizado de maneira adaptada em um estudo, com sessões de 20 minutos. Foi aplicado em uma escola com crianças de 8 a 11 anos, para uma abordagem sobre a asma, de maneira interativa, baseada em discussões em grupo, jogos, teatros, cartazes e folhetos com atividades lúdicas. A fim de envolver o estudante no processo de aprendizagem, esse programa pode ser realizado na escola por qualquer pessoa treinada: enfermeira, funcionários da escola, familiares e voluntários da comunidade ${ }^{(12)}$.

Uma estrutura semelhante foi desenvolvida no estudo que utilizou o Kickin'Asthma, descrito pelos autores como uma adaptação do The Open Airways ao nível cognitivo de adolescentes. No Kickin'Asthma, 4 sessões de 50 minutos foram conduzidas por enfermeiras, por meio de atividades que incluíam jogos, vídeos e teatros, a fim de promover educação sobre a asma. Destaca-se que a primeira sessão consistiu em conhecer como cada estudante enfrentava a asma, frequência dos sintomas, utilização de serviços de saúde, ações de autocuidado e medicamentos utilizados $^{(21)}$.

Também focado na asma, o programa Asthma Plan for Kids foi colocado em prática com escolares da área rural, com 16 sessões de 15 minutos, durante 5 semanas, por meio de jogos, folhetos e atividades práticas, intercalados com apresentações didáticas breves. Os autores constataram que os escolares que participaram das atividades tiveram diminuição nas hospitalizações e consultas médica, devido à redução da gravidade da asma ${ }^{(28)}$.

As atividades lúdicas são positivas, pois possibilitam o desenvolvimento integral do escolar, nos âmbitos social, físico, intelectual, cultural e emocional. Por meio dessas atividades, o escolar forma conceitos, relaciona ideias, faz relações lógicas, desenvolve expressão oral e corporal, proporciona integração e constrói seu próprio conhecimento ${ }^{(37)}$, inclusive sobre questões de saúde.

Por outro lado, há de se considerar que a utilização de métodos lúdicos não garante uma mudança de paradigmas tradicionais de ensino, que desconsideram os saberes e o contexto sociocultural de cada escolar. Exemplo disso pôde ser identificado na pesquisa que realizou atividades educativas sobre a asma na escola, na qual seus autores, apesar de utilizarem materiais lúdicos, como jogos educativos e rodas de conversa, afirmaram que somente no final do estudo perceberam a lacuna, por não terem levado 
em consideração os valores culturais durante as $\operatorname{atividades}^{(19)}$.

Os valores culturais são relevantes, pois crianças e adolescentes carregam para a escola suas vivências, histórias de vida, assim como seus conhecimentos prévios, diretamente influenciados por seus contextos socioculturais, que necessitam ser valorizados e articulados, para que o ensino/aprendizagem ocorram de maneira efetiva ${ }^{(38)}$.

Além dos estudos já mencionados, outro investiu em métodos diferenciados, como a internet ${ }^{(15)}$. Cada vez mais tem ocorrido a aproximação entre o ensino e a internet, tendo em vista o estreito elo existente na atualidade, especialmente entre crianças e adolescentes, com computadores, tablets e celulares.

A pesquisa realizada na Irlanda colocou em prática um programa on-line para apoio a escolares adolescentes asmáticos. Demonstrou ter sido uma experiência positiva, visto que aumentou o conhecimento sobre a patologia ${ }^{(15)}$. Os autores apontaram, ainda, que a tecnologia da informação e comunicação poderia permitir ao adolescente tornar-se mais saudável. Por outro lado, estudos demonstraram que a internet, apesar de bem aceita pelos adolescentes, inclusive quando trata sobre saúde, é menos utilizada por eles, pois temem que muitos sites não sejam confiáveis. Por isso, programas como o implementado no estudo seriam um meio seguro de fornecer informações on-line sobre saúde e proporcionar maior autonomia a esses adolescentes ${ }^{(15)}$.

Outro aspecto relevante observado foram estudos que incluíram não somente os escolares em suas atividades, mas também familiares, professores e comunidade ${ }^{(11,25,31)}$. Exemplo disso, foi o estudo realizado para avaliar um programa de asma escolar ${ }^{(11)}$. Escolares realizaram atividades apoiadas em um livro elaborado com base no programa SHARP, projetado para ser colorido, divertido e educativo. Já os familiares, professores e comunidade foram convidados a participar de um programa no estilo "feira de saúde da asma", a fim de compartilhar informações importantes para os cuidados com o familiar asmático. Após as atividades, constatou-se que houve melhora do conhecimento, gestão, aceitação e controle da asma ${ }^{(11)}$.

Pesquisa também referiu a inclusão de pessoas da comunidade, além dos escolares. No programa de educação sobre a asma, que visou identificar $\mathrm{AAP}^{(25)}$, as sessões educativas realizadas por estudantes de enfermagem incluíram escolares do ensino fundamental, professores e também familiares, para propiciar a continuidade das atividades no espaço da escola ${ }^{(25)}$.

Um programa de educação em saúde bucal, desenvolvido na escola, iniciou com a realização de atividades educativas com os familiares e também treinamento com os professores, para que integrassem o currículo com sessões educativas, e só depois envolveu os estudantes ${ }^{(31)}$.

Pesquisa realizada com adolescentes portadores de alergias alimentares graves, com risco de morte por evento anafilático, forneceu orientações sobre o uso de epinefrina injetável, por meio de fòlderes e vídeos, e fez verificação periódica da disponibilidade do medicamento junto aos escolares. Os autores descreveram que verificar periodicamente a disponibilidade de epinefrina não aumenta a probabilidade de os estudantes terem o medicamento disponível ${ }^{(34)}$. Nesse caso, o contexto sociocultural, que poderia influenciar na decisão e nas possibilidades de acesso ao medicamento, foi ignorado. Essa observância, possivelmente auxiliaria na compreensão de diferentes realidades e, assim, estimularia o autocuidado.

Três estudos sobre saúde bucal realizaram atividades individuais ou em pares com escolares adolescentes ${ }^{(26-27,29)}$. Um deles objetivou avaliar a eficácia de intervenções individuais baseadas no Precaution Adoption Process Model (PAPM) em contraposição às orientações odontológicas tradicionais. Este modelo auxiliou na compreensão de como o adolescente promoveu uma mudança de comportamento e constatou aumento da higiene oral ${ }^{(26)}$.

Os outros dois estudos objetivaram avaliar diferentes intervenções com escolares adolescentes, visando o autocuidado bucal. Um deles realizou intervenções em pares, orientadas pela teoria sociocognitiva, com a participação de um 
dentista $^{(29)}$. A outra pesquisa teve abordagem individual, orientada pela teoria de autoeficácia, foi realizada por um dentista, que iniciou solicitando ao escolar a demonstração do autocuidado oral. Com base nesse relato, fornecia feedback, mostrando deficiências em seu desempenho ${ }^{(27)}$. As duas pesquisas avaliaram os resultados após 6 e 12 meses das intervenções, e ambas concluíram que houve melhoria do autocuidado bucal na avaliação após 6 meses, e descontinuidade de cuidados após 12 meses ${ }^{(27,29)}$.

Outras pesquisas, quando detalharam, referiram utilizar metodologias tradicionais sobre asma $^{(11)}$ e saúde bucal ${ }^{(30)}$. Uma delas, que visava a redução do uso de substâncias nocivas legalizadas, realizou apenas leitura de materiais para os escolares ${ }^{(23)}$. Outros estudos não detalharam como as atividades foram desenvolvidas ${ }^{(19-22,25)}$.

Constatou-se que a utilização do espaço da escola, para a realização e/ou incentivo do autocuidado, tem focado, nos diferentes países, especificamente na doença, e não na saúde, predominantemente por meio de práticas pedagógicas tradicionais. Entretanto, os que incluíam atividades lúdicas e criativas não detalhavam se os contextos socioculturais dos escolares foram levados em consideração para a realização de tais atividades, o que torna questionável se, de fato, estaria ocorrendo mudanças de abordagem, que deixariam de centralizar o saber no profissional, fosse ele da educação ou da saúde.

Não é suficiente a abordagem de questões de autocuidado em saúde, no espaço da escola, por meio de atividades pontuais, descontextualizadas da realidade das crianças e dos adolescentes, que não promovam um olhar crítico e reflexivo dos escolares nem incentivem uma mudança de atitudes no longo prazo.

Há inúmeras falhas no processo de produzir saúde na escola, que geralmente acabam por ser vistas como ações ineficazes. Os profissionais da saúde e/ou educadores geralmente culpabilizam a população pelo não alcance de melhoria de saúde ${ }^{(39)}$, no entanto não percebem que a falha, muitas vezes, não está na população, mas, sim, na maneira de se tentar promover a saúde na escola. Por consequência, tanto a população escolar como os profissionais passam a desacreditar do poder dessa atividade e possivelmente se envolvam cada vez menos com essas ações.

A escola, enquanto instituição, necessita enfrentar seu dever social não somente relacionado às questões de saúde, mas também o de formar cidadãos capazes de articular conhecimentos de maneira crítica, e que utilizem esses saberes para provocar mudanças individuais e coletivas.

\section{Papel da enfermagem no autocuidado escolar}

Não obstante o papel da enfermagem não ter sido o foco da revisão, durante a análise dos estudos pôde-se observar a presença de enfermeiros nas pesquisas, os chamados "enfermeiros escolares"(12,15,19,28,33-34). Alguns estudos sugerem que este profissional deveria estar mais presente nesse contexto, pois compreendem que contribuiriam de maneira significativa para o processo de autocuidado em saúde ${ }^{(15,19,33)}$. Argumentam ainda que enfermeiros de saúde pública têm uma grande responsabilidade na prestação de $\operatorname{serviços~}^{(15)}$.

Com a justificativa de que, na revisão desenvolvida, muitos estudos examinaram o impacto de programas desenvolvidos nas escolas sobre alunos com asma, mas que não fornecem a elaboração adequada dos componentes dos programas, o que torna difícil sua replicação, a pesquisa realizada em Chicago/Illinois (EUA) analisou o processo de gerenciamento de casos de asma pela enfermeira escolar, na prestação de intervenções específicas ${ }^{(19)}$. O estudo demonstrou o impacto do gerenciamento dessas profissionais sobre a saúde das crianças com doenças crônicas, como a asma, para torná-las mais autônomas na gestão de seus próprios sintomas.

No estudo realizado em Minnesota (EUA), os autores destacaram que, reconhecer a enfermeira da escola como membro da equipe de saúde e a escola como um local para o intercâmbio de informações sobre saúde resultaria na prestação de cuidados essenciais na escola, com redução do número de atendimentos de emergência e de 
absenteísmo devido à doença, com consequente melhoria nos resultados de aprendizagem ${ }^{(33)}$.

Compreende-se a relevância da presença de enfermeiros nas escolas, mas sua atuação deve estar pautada em ações de saúde que ultrapassem a concepção biologicista, para que, desta maneira, consiga gerar impactos na saúde do escolar, especialmente no que se refere ao autocuidado em saúde. Pondera-se, no entanto, a grande demanda de trabalho da enfermagem, especialmente em locais em que não há um enfermeiro específico para a escola, assim como o impacto que a formação desse profissional pode ocasionar quando orientada pelo modelo biomédico.

Destaca-se como limitação desta pesquisa a utilização do descritor "Serviços de saúde escolar", definido no PubMed e Descritores em Ciências da Saúde (DeCS) como serviços de saúde preventiva prestados aos estudantes, o que pode ter ocasionado a identificação de estudos que aproximaram, em sua maioria, o autocuidado em saúde na escola a questões biologicistas, deixando de incluir pesquisas que abordaram saúde na escola de maneira ampliada, contextualizada na realidade sociocultural.

Não obstante nenhum estudo incluído ter sido realizado no Brasil, considera-se que a presente revisão integrativa contribui, ao estimular a reflexão crítica de profissionais da saúde e de enfermagem, sobre as experiências expostas em diferentes países. Diante do atual panorama sobre autocuidado em saúde na escola, é preciso que realizem atividades que promovam o autocuidado em saúde na escola de maneira efetiva, por meio de ações que considerem o escolar em seu contexto sociocultural.

\section{Conclusão}

Ações de educação em saúde na escola são historicamente limitadas no que se refere à temática e à metodologia de ensino. Por esta razão, a presente revisão integrativa fornece um panorama sobre as ações de saúde no âmbito do autocuidado atualmente realizadas em escolas, possibilitando identificar lacunas nesse processo em diferentes países e demonstrando que a problemática é global.

As ações de autocuidado em saúde na escola estão ainda atreladas às patologias, especialmente aquelas que ocasionam a ausência dos estudantes na escola e, por consequência, interferem no processo de ensino-aprendizagem. Esta perspectiva é importante, e essas pesquisas têm impacto positivo, porém acredita-se que é necessário ir além do foco na doença, para produzir saúde de maneira efetiva na escola.

Além da temática trabalhada nas escolas, outro ponto que se destacou foi a maneira como essas ações foram realizadas. Diferentes estratégias foram identificadas nos estudos, como programas on-line, jogos, histórias, folhetos, vídeos e discussões, no entanto a questão sociocultural não foi levada em consideração, o que pode impactar na efetividade das ações, especialmente no longo prazo.

O profissional da enfermagem tem papel central nesse contexto, por sua proximidade ao contexto escolar, possuindo potencial para incentivar, de forma efetiva, ações de autocuidado em saúde.

A ausência de produções brasileiras neste estudo indica a necessidade de ampliar a divulgação científica de pesquisas que colocam o enfermeiro como protagonista na promoção da saúde nas escolas do país, especialmente no que se refere ao autocuidado. Dessa forma, o presente estudo fornece contribuições a esses e aos demais profissionais da saúde, incentivando-os para que realizem ações efetivas no espaço escolar.

\section{Colaborações:}

1 - concepção, projeto, análise e interpretação dos dados: Marjoriê da Costa Mendieta, Eliana Buss, Natália Rosiely Costa Vargas e Silvana Ceolin; 
2 - redação do artigo, revisão crítica relevante do conteúdo intelectual: Marjoriê da Costa Mendieta, Eliana Buss, Natália Rosiely Costa Vargas, Silvana Ceolin, Kamila Dias Gonçalves e Rita Maria Heck;

3 - aprovação final da versão a ser publicada: Marjoriê da Costa Mendieta, Kamila Dias Gonçalves e Rita Maria Heck.

\section{Referências}

1. Silva CS, Bodstein RCA. Referencial teórico sobre práticas intersetoriais em Promoção da Saúde na Escola. Ciênc Saúde Coletiva. 2016;21(6):1777-88. DOI: 10.1590/1413-81232015216.08522016

2. Galvão MTRLS, Janeiro JMSV. O autocuidado em enfermagem: autogestão, automonitorização e gestão sintomática com conceitos relacionados. REME Rev Min Enferm. 2013;17(1):213-35. DOI: $10.5935 / 1415-2762.20130019$

3. Carvalho FFB. A saúde vai à escola: a promoção da saúde em práticas pedagógicas. Physis. 2015;25(4):1207-27. DOI: 10.1590/ S0103-73312015000400009

4. Antunes LS, Antunes LAA, Corvino MPF. Educative practices and attitudes within the pre-school environment: evaluating the education professionals. Braz Oral Res. 2008 Dec;22(4):340-5. DOI: $10.1590 /$ S1806-83242008000400010

5. Leal LB, Pereira KLA, Negreiros ALB, Pequeno AMC, Lima GP, Negreiros FDS, et al. Método ativo problematizador como estratégia para formação em saúde. Rev Enferm UFPE on line. 2018 abr;12(4):1139-43. DOI: 10.5205/1981-8963-v12i4a 231346p1139-1143-2018

6. Brasil. Ministério da Saúde. Secretaria de Gestão do Trabalho e de Educação na Saúde. A educação que produz saúde [Internet]. Brasília, DF; 2005 [cited 2019 May 12]. Available from: http://bvsms. saude.gov.br/bvs/publicacoes/educacao_que_ produz_saude.pdf

7. Ercole FF, Melo LS, Alcoforado CLGC. Revisão integrativa versus revisão sistemática. REME rev min enferm. 2014;18(1):9-11. DOI: $10.5935 / 1415-2762.20140001$

8. Mendes KDS, Silveira RCCP, Galvão CM. Revisão integrativa: método de pesquisa para a incorporação de evidências na saúde e na enfermagem. Texto
Contexto Enferm. 2008;17(4):758-64. DOI: 10.1590/ S0104-07072008000400018

9. Moher D, Liberati A, Tetzlaff J, Altman DG. Principais itens para relatar revisões sistemáticas e meta-análises: a recomendação PRISMA. Epidemiol Serv Saúde. 2015;24(2):335-42. DOI: $10.5123 /$ S1679-49742015000200017

10. Bixo OA, Akesson K, Ilvered R, Forsander G, Särnblad S. Self-care management of type 1 diabetes has improved in Swedish schools according to children and adolescents. Acta paediatr. 2017 Dec;106(12):1987-93. DOI: 10.1111/apa.13949

11. Kintner EK, Cook G, Marti CN, Allen A, Stoddard D, Harmon P, et al. Effectiveness of a school-and community-based academic asthma health education program on use of effective asthma selfcare behaviors in older school-age students. J Spec Pediatr Nurs. 2015;20(1):62-75. DOI: 10.1111/ jspn.12099

12. Crane LM, O'neal KS, Honey BL, Kirkpatrick A. Effectiveness of a modified open airways curriculum. J Asthma. 2015 Jun;52(5):519-27. DOI: 10.3109/02770903.2014.986739

13. Gefter L, Rosas LG, Rodrigues E, MoriokaDouglas N. Training at-risk youth to become diabetes self-management coaches for family members. Diabetes Educ. 2014;40(6):786-96. DOI: $10.1177 \% 2 F 0145721714549676$

14. Guo JL, Liao JY, Chang LC, Wu H, Huang CM. The effectiveness of an integrated multicomponent program for adolescent smoking cessation in Taiwan. Addict Behav. 2014;39(10):1491-9. DOI: 10.1016/j.addbeh.2014.05.009

15. Hughes M, Murphy M. Evaluation of a pilot national online asthma E-learning program for secondary school students. Compr Child Adolesc Nurs. 2014;37(2):136-46. DOI: 10.3109/01460862.2014.902405

16. Särnblad S, Berg L, Detlofsson I, Jönsson A, Forsander G. Diabetes management in Swedish schools: a national survey of attitudes of parents, children, and diabetes teams. Pediatr Diabetes. 2014;15(8):550-6. DOI: 10.1111/pedi.12133

17. Tang SM, Chen SW, Wang RH. Establishing a model to assess the effects of school support and self-care behaviors on life satisfaction in adolescents with type 1 diabetes in Taiwan. J Nurs Res. 2013;21(4):24451. DOI: 10.1097 jnr.0000000000000008

18. Engelke MK, Swanson M, Guttu M. Process and outcomes of school nurse case management for 
students with asthma. J Sch Nurs. 2014;30(3):196205. DOI: $10.1177 \% 2$ F1059840513507084

19. Velsor-Friedrich B, Militello LK, Richards MH, Harrison PR, Gross IM, Romero E, et al. Effects of coping-skills training in low-income urban African-American adolescents with asthma. J Asthma. 2012 May;49(4):372-9. DOI: 10.3109/02770903.2012.660296

20. Al-Sheyab N, Gallagher R, Crispm J, Shah S. Peer-led education for adolescents with asthma in jordan: a cluster-randomized controlled trial. Pediatrics. 2012 Jan;129(1):106-12. DOI: 10.1542/ peds.2011-0346

21. Shrimali BP, Hasenbush A, Davis A, Tager I, Magzame S. Medication use patterns among urban youth participating in school-based asthma education. J Urban Health. 2011 Feb;88(1):73-84. DOI: $10.1007 /$ s11524-010-9475-Z

22. Melnyk BM, Jacobson D, Kelly S, O'haver J, Small L, Mays MZ. Improving the mental health, healthy lifestyle choices, and physical health of hispanic adolescents: a randomized controlled pilot study. J Sch Health. 2009 Dec;79(12):575-84. DOI: 10.1111/j.1746-1561.2009.00451.x

23. Gruenewald PJ, Johnson K, Shamblen SR, Ogilvie KA, Collins D. Reducing adolescent use of harmful legal products: intermediate effects of a community prevention intervention. Subst Use Misuse. 2009;44(14):2080-98. DOI: $10.3109 / 10826080902855223$

24. Johnston CA, Tyler C, Mcfarlin BK, Poston WSC, Haddock CK, Reeves RS, Foreyt JP. Effects of a school-based weight maintenance program for mexican-american children: results at 2 years. Obesity. 2010 Mar;18(3):542-7. DOI: 10.1038/ oby.2009.241

25. McClure N, Seibert M, Johnson T, Kannenberg $\mathrm{L}$, Brown $\mathrm{T}$, Lutenbacher M. Improving asthma management in the elementary school setting: an education and self-management pilot project. J Pediatr Nurs. 2018 Sep-Oct;42:16-20. DOI: 10.1016/j.pedn.2018.06.001

26. Aleksejūnienė J, Brukienė V. A cluster randomized theory-guided oral hygiene trial in adolescents-A latent growth model. Int J Dent Hyg. 2017;16(2):e23e30. DOI: /10.1111/idh.12286

27. Džiaugytė L, Aleksejūnienė J, Brukienè V, Pečiulienè V. Self-efficacy theory-based intervention in adolescents: a cluster randomized trial-focus on oral self-care practice and oral self-care skills. Int
J Paediatr Dent. 2017;22(1):37-46. DOI: 10.1111/ ipd. 12223

28. Horner SD, Brown A, Brown SA, Rew DL. Enhancing asthma self-management in rural school-aged children: a randomized controlled trial. J Rural Health. 2016;32(3):260-8. DOI: 10.1111/jrh.12150

29. Aleksejūnienė J, Brukienė V, Džiaugyte L, Pečiulienė V, Bendinskaitè R. A theory-guided school-based intervention in order to improve adolescents' oral self-care: a cluster randomized trial. Int J Paediatr Dent. 2016;26(2):100-9. DOI: 10.1111/ipd.12164

30. Pakpour AH, Yekaninejad MS, Sniehotta FF, Updegraff JA, Dombrowski SU. The effectiveness of gain-versus loss-framed health messages in improving oral health in Iranian secondary schools: a cluster-randomized controlled trial. Ann Behav Med. 2014;47(3):376-87. DOI: 10.1007/ s12160-013-9543-1

31. Esan A, Folayan MO, Egbetade GO, Oyedele TA. Effect of a school-based oral health education programme on use of recommended oral selfcare for reducing the risk of caries by children in Nigeria. Int J Paediatr Dent. 2015;25(4):282-90. DOI: $10.1111 /$ ipd.12143

32. Velsor-Friedrich B, Militello LK, Kouba J, Harrison PR, Manion A, Doumit R. Pediatric obesity and asthma quality of life. Nurs Clin North Am. 2013;48(2):259-70. DOI: 10.1016/j.cnur.2013.01.011

33. Hanson TK, Aleman M, Hart L, Yawn B. Increasing availability to and ascertaining value of asthma action plans in schools through use of technology and community collaboration. J Sch Health. 2013;83(12):915-20. DOI: doi.org/10.1111/ josh. 12110

34. Spina JL, McIntyre CL, Pulcini JA. An intervention to increase high school students' compliance with carrying auto-injectable epinephrine: a MASNRN study. J Sch Health. 2012;28(3):230-7. DOI: $10.1177 / 1059840511431459$

35. Stillwell SB, Fineout-Overholt E, Melnyk BM, Williamson KM. Evidence-based practice, step by step: searching for the evidence. Am J Nurs, 2010 May;110(5):41-7. DOI: 10.1097/01. NAJ.0000372071.24134.7e

36. Santos RM, Gomes NP, Mota RS, Gomes NP, Couto TM, Araújo GS. Reprovação escolar e aspectos sociais e de saúde: estudo transversal com adolescentes. Rev baiana enferm. 2017;32:e21827. DOI: $10.18471 /$ rbe.v32.21827 
37. Santos SMP. O lúdico na formação do educador. 9a ed. Petrópolis: Vozes; 2011.

38. Freire P. Educação como prática de liberdade. 40a ed. Rio de Janeiro: Paz e Terra; 2017.

39. Casemiro JP, Fonseca ABC, Secco FVM. Promover saúde na escola: reflexões a partir de uma revisão sobre saúde escolar na América Latina. Ciênc Saúde Coletiva. 2014;19(3):829-40. DOI: 10.1590/1413-81232014193.00442013

Recebido: 29 de julho de 2019

Aprovado: 18 de outubro de 2019

Publicado: 19 de dezembro de 2019

A Revista Baiana de Enfermagem utiliza a Licença Creative Commons - Atribuição-NãoComercial 4.0 Internacional.

https://creativecommons.org/licenses/by-nc/4.0/

Este artigo é de acesso aberto distribuído sob os termos da Licença Creative Commons (CC BY-NC).

Esta licença permite que outros remixem, adaptem e criem a partir do seu trabalho para fins não comerciais. Embora os novos trabalhos tenham de lhe atribuir o devido crédito e não possam ser usados para fins comerciais, os usuários não têm de licenciar esses trabalhos derivados sob os mesmos termos. 